05

\title{
О разрушении упругих полимерных материалов под воздействием электронного пучка
}

\author{
() М.И. Гуревич, Е.Д. Казаков, Ю.Г. Калинин, А.А. Курило, О.В. Тельковская, К.В. Чукбар \\ Национальный исследовательский центр „Курчатовский институт“, \\ 123098 Москва, Россия \\ e-mail: chukbar_kv@nrcki.ru
}

Поступило в Редакцию 16 декабря 2020 г.

В окончательной редакции 31 мая 2021 r.

Принято к публикации 2 июня 2021 г.

Предложено объяснение обнаруженной в нескольких экспериментах особенности в общей картине разрушения нехрупких полимеров под воздействием ударной волны, инициируемой мощным электронным пучком. Удаленность области растрескивания от поверхности материала, на которую воздействует пучок, на конечное расстояние в глубину связывается с трехмерным характером распространения упругих волн. Универсальность эффекта продемонстрирована на простейшей изотропной модели, показывающей, что большие растягивающие напряжения эффективно генерируются внутри мишени при ее достаточно больших поперечных и продольных размерах даже без учета нелинейных и сдвиговых процессов.

Ключевые слова: сильноточные электронные пучки, ударные волны, полимерные материалы, высокие давления.

DOI: 10.21883/JTF.2021.11.51525.346-20

\section{Введение}

Классическими инструментами возбуждения ударных волн в исследованиях в области физики высоких динамических давлений являются взрывные и баллистические генераторы. Стремление к дальнейшему продвижению в область недоступных ранее параметров и возможности организации ударно-волновых исследований в условиях обычной физической лаборатории побудило использовать другие способы интенсивного воздействия на вещество. В частности, в качестве источников высоких динамических давлений для исследований упругопластических и прочностных свойств металлов и пластиков широко применяются импульсные лазеры большой мощности и сильноточные электронные пучки (см., например, [1-4]).

Ранее в экспериментальных исследованиях воздействия мощного импульсного электронного пучка на мишени из полимерных материалов, в частности оргстекла (полиметилметакрилата - ПММА) и эпоксидной смолы (ЭДП), проведенных на сильноточном генераторе релятивистских электронных пучков (РЭП) „Кальмар“ [1,2], была зафиксирована „нестандартная“ картина их разрушений. В рассматриваемых экспериментах максимальная энергия электронов пучка варьировалась в диапазоне $200-300 \mathrm{keV}$, максимальный ток пучка составлял от 10 до $20 \mathrm{kA}$ при длительности импульса на полувысоте $100 \mathrm{~ns}$. В условиях, когда фронтальные размеры мишени в несколько раз превышают диаметр пятна взаимодействия пучка с ее поверхностью, а толщина образца вполне с ним сопоставима или больше, между областью выделения энергии РЭП и областью внутреннего повреждения (растрескивания) полимера остается прозрачная зона незатронутого эрозией материала размером по направлению распространения пучка 2-6 mm при диаметре пучка $10-20 \mathrm{~mm}$. В указанном диапазоне параметров толщина этой зоны практически не зависит от геометрических характеристик облучаемого образца и определяется только параметрами падающего электронного пучка. В частности, при увеличении диаметра пучка на поверхности мишени толщина прозрачной зоны увеличивается. Этим картина повреждений упомянутых полимеров отличается от подобной для материалов, подверженных „хрупкому“ разрушению, например, стекла или полистирола $[1,2]$. Возможные причины существенного отличия в „хрупкости“ для полимеров могут быть связаны с особенностями релаксации напряжений и более подробно рассмотрены в работах $[5,6]$.

Причина описанного эффекта до сих пор не установлена, хотя среди каких процессов ее следует искать, было осознано сразу. Глубина энерговклада пучка имеет порядок сотен микрон, и потому разрушения в объеме и на тыльной стороне образцов возникают под воздействием генерируемого нагревом от РЭП упругого возмущения материала, распространяющегося по мишени и имеющего на переднем фронте характер ударной волны. Собственно, именно инициированию таких волн и были посвящены работы [1-4].

Типичная постановка обсуждаемых экспериментов описана ниже (рис. 1).

Образец полимера в форме прямоугольного параллелепипеда помещался в анодный узел диода генератора „Кальмар“, в котором при подаче высоковольтного импульса на катод за счет взрывной эмиссии формировался РЭП, облучающий образец. Радиальное распределение вклада энергии РЭП в мишень определялось по рентге- 


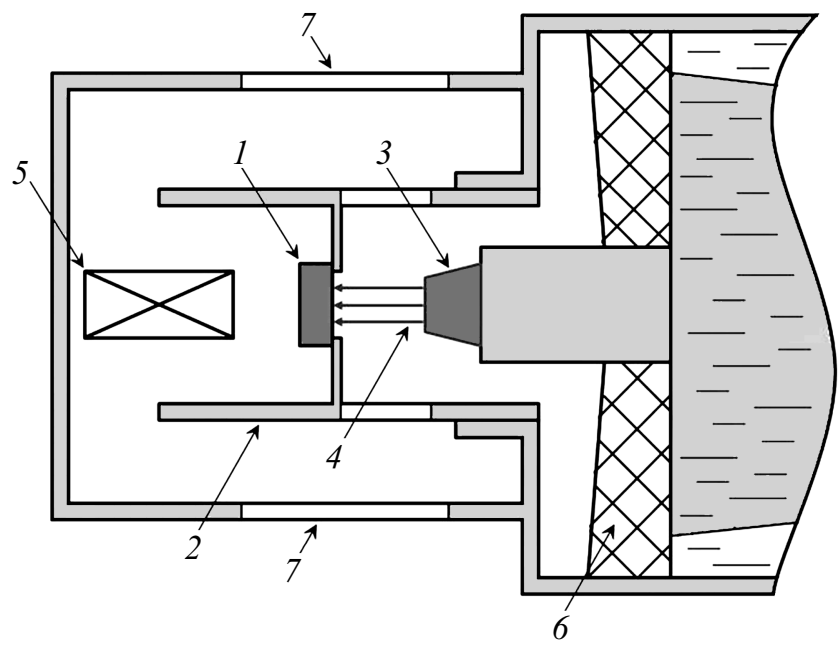

Pис. 1. Схема экспериментов по изучению воздействия РЭП на полимерные материалы: 1 - исследуемый образец, 2 - анодный узел, 3 - катод, 4 - РЭП, 5 - камера-обскура, 6 - изолятор диода, 7 - смотровые окна.

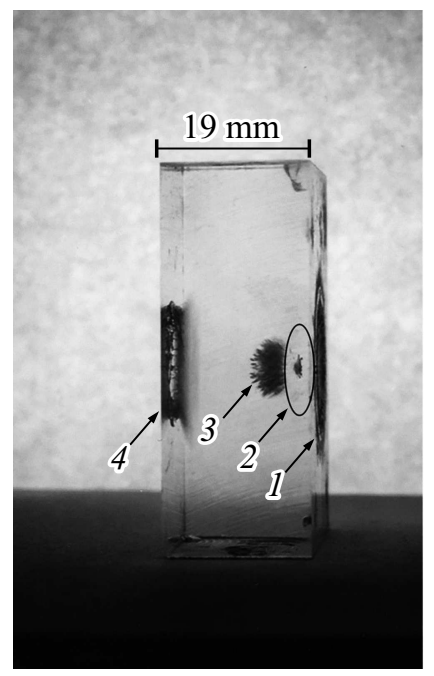

Рис. 2. Образцы ПММА размером $5 \times 5 \mathrm{~cm}(a)$ и ЭДП размером $6.2 \times 6.2 \mathrm{~cm}(b)$, облученные электронным пучком; РЭП падал на правую поверхность образцов. Цифрами обозначены: 1 - зона испарения и оплавления материала мишени под действием электронного пучка, 2 - прозрачная зона, 3 - зона внутреннего разрушения, 4 - „откольная“ зона, возникающая при отражении ударной волны от этой границы.

новским фотографиям, полученным с помощью рентгеновской камеры обскуры, расположенной за образцом. Стандартно форма этого распределения была близка к гауссовой. Особенности проведения экспериментов на ускорителе „Кальмар“ и техника измерений параметров пучка подробно изложены в работах $[1,2]$. На рис. 2 представлены фотографии образцов ПММА и эпоксидной смолы (ЭДП) после воздействия на них электронного пучка; в дальнейшем подобные эффекты наблюдались неоднократно (см., например, $[7,8]$ ).

Хорошо известно, что разрушение упруго-пластичных материалов при растяжении наступает при абсолютных значениях механического напряжения, заметно (в разы) меньших, чем при сжатии. В рамках изучения обнаруженного явления на той же установке „Кальмар“ были проведены эксперименты по установлению момента разрушения ПММА при наблюдении распространения по образцу ударной волны, инициированной РЭП, с хорошим временным разрешением [7]. Оказалось, что эрозия материала происходит при его „разгрузке“ уже после прохождения фронта ударной волны, т.е. как раз на стадии растяжения пластика.

Для сравнения на рис. 3 приведена фотография разрушенного образца полистирола, облученного электронным пучком при сходных условиях. Видно, что никакой прозрачной зоны между областью воздействия электронного пучка на поверхность образца и разрушения не наблюдается.

В настоящей работе, опираясь на указанные выше особенности явления, предлагается возможное объяснение эффекта „нестандартной“ картины разрушения — наличия прозрачной области между зонами энерговыделения и разрушения, связанное со спецификой трехмерной эволюции упругой волны, возбуждаемой импульсом давления на границе среды. Существенно, что обсуждаемый далее эффект появления растягивающих напряжений на

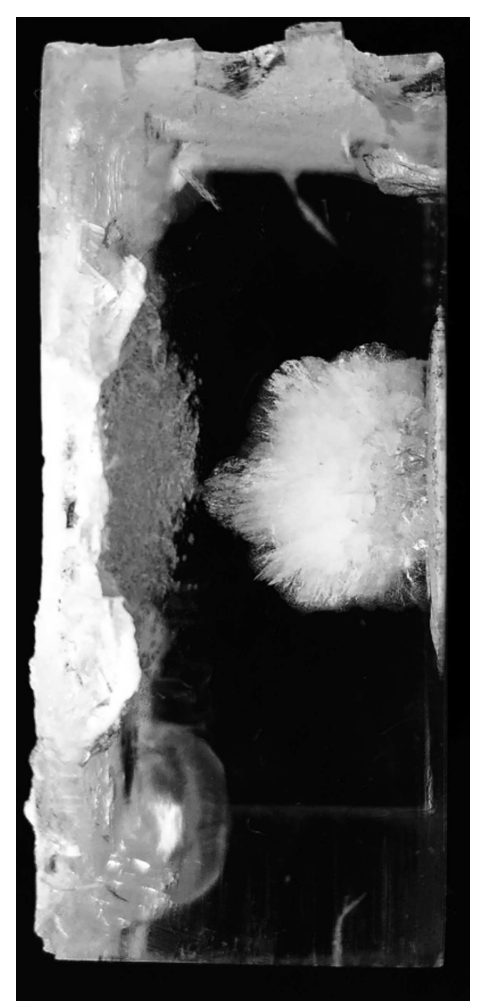

Рис. 3. Образец полистирола размером $5.2 \times 5.2 \mathrm{~cm}$ толщиной $2.3 \mathrm{~cm}$, облученный электронным пучком; РЭП падал на правую поверхность образца. 
удалении от поверхности мишени носит чрезвычайно общий характер, не зависящий от вариаций механических свойств материала. По этой причине изложение не концентрируется на конкретной структуре полимера, от которой, естественно, зависят эти свойства и даже возможна анизотропия.

\section{1. Физическая модель}

Распространение упругих волн, как известно, можно достаточно эффективно представить на основе простой акустической модели, согласно которой эволюция напряжения в среде $\sigma$ описывается линейным волновым уравнением с постоянной скоростью звука $c_{S}$ :

$$
\frac{\partial^{2} \sigma}{\partial t^{2}}-c_{S}^{2} \Delta \sigma=0
$$

$[9,10]$. В данном случае такое приближение оправдано тем, что достигаемые в эксперименте значения $\sigma$, с одной стороны, не слишком велики, и наблюдаемые скорости ударных волн не сильно превышают так называемую объемную скорость звука (см. ниже), а с другой - достаточны для преодоления предела текучести пластика, когда упругие свойства материала становятся аналогичными свойствам жидкости или газа (за исключением того, что напряжение - оно же давление - может принимать и отрицательные значения). Соответственно скорость звука в (1) имеет порядок $\sqrt{K / \rho}$, где $K-$ модуль объемного сжатия материала, а $\rho-$ его плотность, в то время как модуль сдвига $G \rightarrow 0[9,12]$. Кроме того, простота модели делает выводы на ее основе особенно наглядными и надежными. Эффекты нелинейности, анизотропии и диссипации, конечно, способны модифицировать некоторые количественные характеристики упругих процессов, но не могут поменять декларируемой качественной картины явления. Далее процесс в рамках (1) рассматривается в цилиндрической системе координат с осью $z$, направленной от границы, на которую попадает РЭП, вглубь образца. Поскольку профиль этого пучка можно считать аксиально-симметричным, $\sigma$ в (1) является функцией $t, z$ и $r$, но не $\varphi$.

Объяснению подлежит не само наличие растягивающих напряжений $\sigma<0$, а достижение ими достаточных для разрушения материала значений лишь с удалением возмущения от границы на конечное расстояние. В [7], где было экспериментально доказано растрескивание оргстекла именно при его растяжении, возникновение области $\sigma<0$ теоретически моделировалось по стандартной схеме [9] на основе одномерного (по $z$, т.е. при $\sigma=\sigma(t, z))$ акустического уравнения, когда неограниченный и однородный по $r$ (напомним, в цилиндрической системе координат) электронный пучок быстро нагревает приповерхностный слой материала, порождая тем самым возмущение напряжения в среде, распространяющееся далее согласно (1). В этом случае, поскольку пучок вносит в среду энергию, но не импульс, ${ }^{1}$ у формирующейся упругой волны, бегущей вглубь образца, практически сразу (на расстояниях порядка толщины области энерговклада, составляющей, как указано выше, всего несколько сотен микрон) появляются компенсирующие друг друга участки с $\sigma>0$ в голове и $\sigma<0$ (за счет так называемой „разгрузки“) на хвосте [7,9] только в этом случае среда будет обладать нулевым полным импульсом $\int \rho \mathbf{v} d z$, где $\mathbf{v}-$ массовая скорость среды. С удалением от границы амплитуда возмущения в реальности уменьшается из-за неучтенных эффектов диссипации и расхождения волны по радиусу вследствие конечной толщины пучка, ее породившего, так что максимум растягивающих напряжений в рамках такой модели должен достигаться вблизи границы, что не соответствует наблюдаемой картине „оторванности“ области растрескивания (рис. 2).

В настоящей работе мы обращаем внимание на то, что эту картину можно объяснить, изменив модельное представление о механизме воздействия пучка на образец и представление о геометрии задачи. Сами изменения отнюдь не оригинальны (см. ссылки далее), но интересно, что к цели ведет учет всего двух модификаций. За достижение больших отрицательных значений $\sigma$ лишь в глубине материала ответствен комбинированный эффект испарения и разлета вещества образца в области энерговклада РЭП вкупе с ограниченностью возбуждаемой упругой волны по всем трем пространственным измерениям, т.е. вследствие ее трехмерного характера. Последнее обстоятельство в рамках совсем другой модели предлагалось учитывать еще в [2].

Действительно, повреждения экспериментальных образцов и наблюдения разлета плазмы с облучаемой поверхности [13] свидетельствуют о том, что нагрев их РЭП не просто создает упругое напряжение в приповерхностном слое, но испаряет (и ионизует) его, после чего этот слой начинает неограниченно разлетаться, не испытывая влияния возвратной упругой силы, наличествующей в нагретом, но остающемся твердым материале в модели [7,9]. Такой разлет оказывает длительное в сравнении со временем воздействия пучка давление на незатронутые испарением слои материала за счет реактивной силы, внося в конденсированную среду уже вполне заметный импульс, обнаруживаемый экспериментально [14]. Иными словами, рождение упругого возмущения в мишени в рамках (1) в области $z>0$ адекватно описывается заданием на границе $z=0$ импульса положительного давления. В одномерной ситуации возникающая при этом акустическая волна обладала бы исключительно неотрицательными значениями $\sigma$. Однако, поскольку в реальности, как подчеркнуто во Введении, область энерговклада РЭП в поперечнике заметно меньше размера образца, эта волна вполне трехмерна, а хорошо известно (см., например, $[15,16])$,

\footnotetext{
${ }^{1}$ Конечно, поглощаемые электроны обладают и импульсом, но его влияние в силу их незначительной массы пренебрежимо мало.
} 
что в высших размерностях в отличие от одномерии в рамках (1) монополярная волна с $\sigma \geq 0$ существовать не может. Этот факт обычно поясняется следующим соображением. Наличие напряжения в линейной изотропной среде приводит ее в движение согласно второму закону Ньютона $\rho \partial \mathbf{v} / \partial t=-\nabla \sigma)$. Скорость среды в акустическом приближении потенциальна $\mathbf{v}=-\nabla \theta$, так что получается связь $\int \sigma d t \propto \theta$. Если в точке наблюдения до и после прохождения через нее звуковой волны среда находится в покое, характеризуемом нулевым потенциалом, то, значит, в любой локализованной волне обязательно присутствуют участки с $\sigma$ обоих знаков иначе интеграл по времени не будет обнуляться. Одномерная волна разделяет пространство на области перед и за ней, и значения $\theta$ на $\pm \infty$ могут различаться, но при локализации волны по всем направлениям область покоя вне ее связна и потому характеризуется одним значением потенциала. Таким образом, если реактивное давление исходно создает в упругом образце квазиодномерную (ибо ее размер по $r$ значительно превосходит толщину по $z$, см. далее) волну сжатия, то с ее удалением от границы на расстояния, сопоставимые с ее же поперечным размером, в игру вступает трехмерность, что приводит к „генерации“ участков растяжения.

Отметим, что эффект достижения максимального растяжения материала в глубине мишени наблюдался в расчетах [4], связанных с возбуждением ударных волн в стальных образцах. Однако механизм этого явления был другим, область с $\sigma<0$ исходно появлялась на краю пучка и на границе мишени, а последующий рост амплитуды возмущения происходил при схождении волны от края к оси. Скорее всего, это было связано все же с разгрузкой при неполном испарении стали пучком и тем, что в области возникновения растяжения $G \neq 0$ (описание расчетов не слишком подробно). Наш механизм представляется более универсальным.

\section{2. Аналитические и численные расчеты}

Перейдем к строгим выкладкам. Требуется найти решение (1) $\sigma(t, \mathbf{r}, z)$, где $\mathbf{r}$ - двумерный вектор в плоскости $x y$, т. е. $\sigma(t, r, \varphi, z)=\sigma(t, r, z)$ в силу указанной выше симметрии задачи, в области $z>0$ при заданных начальных $\sigma=0, \partial \sigma / \partial t=0$ при $t=0$ и граничном, которое для упрощения формул, записывается в виде функции с разделяющимися переменными, $\sigma=g(t) P(r)$ при $z=0$ условиях. Конкретные профили $g(t)$ и $P(r)$ будут заданы ниже. В силу линейности задачи, это решение выражается сверткой граничного условия с некоторой характеризующей $(1)$ функцией $L(t, r, z)$, которая и подлежит определению. Действительно, проводя преобразования Лапласа по $t$ и двумерное Фурье по $\mathbf{r}$ и пользуясь тем, что для них $\partial^{2} / \partial t^{2} \rightarrow p^{2}, \Delta \rightarrow-k^{2}+\partial^{2} / \partial z^{2}$, можно написать, что

$$
\frac{d^{2} \sigma_{p \mathbf{k}}}{d z^{2}}=\left(\frac{p^{2}}{c_{S}^{2}}+k^{2}\right) \sigma_{p \mathbf{k}} \rightarrow \sigma_{p \mathbf{k}}=g_{p} P_{\mathbf{k}} L_{p \mathbf{k}},
$$

где

$$
L_{p \mathbf{k}}=\exp \left[-z \sqrt{\left(p / c_{S}\right)^{2}+k^{2}}\right] .
$$

Здесь использовано стандартное добавление нижних индексов $p$ для лаплас- и $\mathbf{k}$ для фурье-образов соответствующих функций (см., например, [17]). Обращая преобразование Фурье, получаем

$$
\begin{aligned}
& L_{p}=\frac{1}{2 \pi} \int_{0}^{\infty} \exp \left[-z \sqrt{\left(p / c_{S}\right)^{2}+k^{2}}\right] J_{0}(k r) k d k \\
& =\frac{z}{2 \pi\left(r^{2}+z^{2}\right)^{3 / 2}}\left(1+\frac{p \sqrt{r^{2}+z^{2}}}{c_{S}}\right) \exp \left(-\frac{p \sqrt{r^{2}+z^{2}}}{c_{S}}\right),
\end{aligned}
$$

$\left(J_{0}(\alpha)=\int_{0}^{\pi} \exp (i \alpha \cos ) d \varphi / \pi-\right.$ функция Бесселя) [18].

Отсюда следует, что

$$
\begin{aligned}
& \sigma(t, r, z)=\int \frac{z P\left(\mathbf{r}-\mathbf{r}_{1}\right)}{2 \pi c_{S}\left(r_{1}^{2}+z^{2}\right)} \\
& \times\left(\frac{c_{S}}{\sqrt{r_{1}^{2}+z^{2}}}+\frac{d}{d t}\right) g\left(t-\sqrt{r_{1}^{2}+z^{2}} / c_{S}\right) d^{2} \mathbf{r}_{1} .
\end{aligned}
$$

Явно видны члены, ответственные за монополярную $(\propto g \geq 0)$ и знакопеременную $(\propto \dot{g})$ слагаемые акустической волны. К сожалению, аналитически этот интеграл удается взять только в двух крайних ситуациях: одномерной с $P=$ const, где $\sigma \propto g\left(t-z / c_{S}\right)$, как и должно быть (знакопеременный член „гасится“ интерференцией), и предельно трехмерной, когда реактивное давление дельта-функционально $P \propto \delta(\mathbf{r})$,

$$
\sigma \propto \frac{\left(\mathbf{e}_{z} \cdot \mathbf{R}\right)}{R^{2}}\left[\frac{g\left(t-R / c_{S}\right)}{R}+\frac{\dot{g}\left(t-R / c_{S}\right)}{c_{S}}\right],
$$

где $\mathbf{R}=\{x, y, z\}-3$-d радиус-вектор, а $\mathbf{e}_{z}-$ единичный вектор (орт) оси аппликат. Здесь монополярное слагаемое представляет собой так называемое „ближнее поле“ точечного акустического источника на границе, а знакопеременное - стандартную дипольную волну $[15,16]$.

Тем не менее найденное интегральное представление является удобным инструментом для моделирования волны, так как численный расчет интегралов существенно надежнее и устойчивее решения дифференциальных уравнений. Для этих целей свертку удобно переписать в виде

$$
\begin{gathered}
\sigma=-\int_{0}^{\infty}\left[\int_{0}^{2 \pi} P\left(\sqrt{r^{2}+r_{1}^{2}-2 r r_{1} \cos \varphi_{1}}\right) d \varphi_{1}\right] \\
\times \frac{z}{2 \pi} \frac{\partial}{\partial r_{1}} \frac{g\left(t-\sqrt{r_{1}^{2}+z^{2}} / c_{S}\right)}{\sqrt{r_{1}^{2}+z^{2}}} d r_{1} .
\end{gathered}
$$




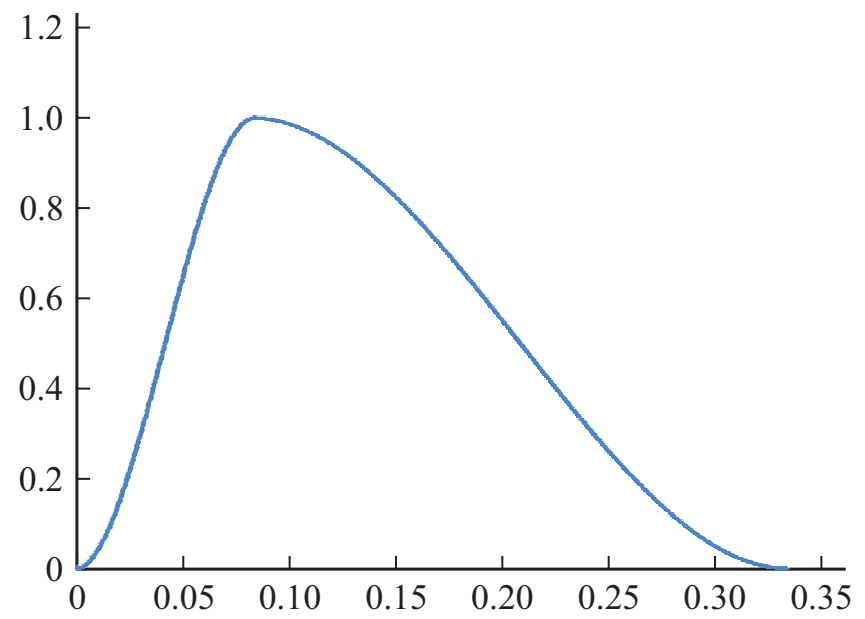

Рис. 4. Зависимость импульса давления на границе от времени в единицах $\vartheta / c_{S}$ для основного варианта расчетов.

Расчет требует задания граничного условия в виде вполне конкретной функции $\sigma(t, r, 0)$. Исходя из указанных во Введении экспериментальных фактов, мы выбрали для нее гауссову пространственную зависимость $P=P_{0} \exp \left(-r^{2} / 2 \vartheta^{2}\right)$ с $\vartheta \sim 3.5 \mathrm{~mm}$. Форма временного импульса давления $g(t)$ представлена на рис. 4.

Это сплайн общей длительности $\tau$, гладко „сшитый“ из двух кубических полиномов, время нарастания которого, по физике дела сопоставимое с длительностью воздействия пучка на образец, было взято в три раза меньше времени спада, связанного с уменьшением давления в реактивной струе вследствие ее необратимого расширения. Изменение соотношения этих времен практически не сказывалось на характере процесса. Вследствие линейности задачи, наблюдаемая в счете картина зависит только от одного безразмерного параметра $c_{S} \tau / \vartheta$, равного отношению продольного и поперечного масштабов возбуждаемой акустической волны (см. выше). Расчет велся в безразмерном виде с $c_{S}=P_{0}=\vartheta=1$, так что единственным варьируемым параметром было $\tau$. Чем оно меньше, тем ближе к одномерии порождаемая волна (в физических переменных уменьшение $\tau$ эквивалентно увеличению $\vartheta$ ), и область отрицательных значений в ней возникает и растет по размерам (с одновременным увеличением здесь и абсолютных значений $\sigma$ ) на задней по отношению к направлению ее распространения стороне профиля на большем удалении от границы. Для демонстрации декларируемого эффекта мы выбрали $(\tau=1 / 3$ : с учетом того, что объемная скорость звука в оргстекле $\sim 2.2 \mathrm{~km} / \mathrm{s}$ [11], это соответствует $\tau \sim 500 \mathrm{~ns}$ в физических переменнных, что находится в хорошем соответствии с наблюдаемой в эксперименте шириной бегущего по образцу возмущения $[7,12]$.

При проведении вычислений обезразмеренная формула (3) с помощью замены независимой $u^{2}=r_{1}^{2}+z^{2}$ и за- висимой $P(s)=F\left(s^{2}\right)$ переменных записывалось в виде

$$
\begin{aligned}
\sigma= & -\int_{0}^{\infty}\left[\int_{0}^{2 \pi} F\left(r^{2}+u^{2}-z^{2}-2 r \sqrt{u^{2}-z^{2}} \cos \varphi_{1}\right) d \varphi_{1}\right] \\
& \times \frac{z}{2 \pi} \frac{\partial}{\partial r_{1}} \frac{g\left(t-u\left(r_{1}\right)\right)}{u\left(r_{1}\right)} d r_{1} \\
= & \int_{z}^{\infty}\left[\int_{0}^{2 \pi} F\left(r^{2}+u^{2}-z^{2}-2 r \sqrt{u^{2}-z^{2}} \cos \varphi_{1}\right) d \varphi_{1}\right] \\
& \times \frac{z}{2 \pi} \frac{g(t-u)+u g^{\prime}(t-u)}{u^{2}} d u .
\end{aligned}
$$

Поскольку вне отрезка $[0, \tau]$ функция $g$ тождественно равна нулю, фактически внешний интеграл считается в пределах $[\max (z, t-\tau), t]$. Кроме того, в силу симметрии аргумента фунции $F$ во внутреннем интеграле расчет велся не для отрезка $[0,2 \pi]$, а $[0, \pi]$. Таким образом, получалась формула

$$
\begin{aligned}
\sigma & =\frac{z}{\pi} \int_{\max (z, t-\tau)}^{t} \\
& \times\left[\int_{0}^{\pi} F\left(r^{2}+u^{2}-z^{2}-2 r \sqrt{u^{2}-z^{2}} \cos \varphi_{1}\right) d \varphi_{1}\right] \\
& \times \frac{g(t-u)+u g^{\prime}(t-u)}{u^{2}} d u .
\end{aligned}
$$

По ней в прямоугольнике $\{0 \leq r \leq 2.8,0 \leq z \leq 2.1\}$ рассчитывалась матрица значений $\sigma\left(r_{i}, z_{j}\right)$ для $r_{i}=0.004 i, 0 \leq i \leq 700$ и $z_{j}=0.004 j, 0 \leq j \leq 525$.

Результаты этих вычислений для трех последовательных моментов времени: сразу по окончании импульса давления и при последующем распространении вглубь среды представлены на рис. 5 .

То, что на картинках имеются точки, для которых волна еще не дошла, не сказывается на времени вычисления, так как в этом случае пределы внешнего интеграла в (4) сразу показывают, что точку рассчитывать не следует. Видно, что область растяжения в упругом возмущении от пучка действительно возникает на конечном удалении от границы (на уровне -0.1 от $\sigma_{\max }$ не ближе $\vartheta$ ). Эта область в сравнении с областью сжатия характеризуется меньшими абсолютными значениями $\sigma$ (что неудивительно при данном механизме генерации возмущения) и меньшими же поперечными размерами. Последнее также находится в хорошем соответствии с реальной картиной разрушения. Ослабление волны, приводящее к ограниченности наблюдаемой области разрушения по $z$ с двух сторон, по-видимому, связано с неучтенной в (1) диссипацией (все-таки на переднем фронте это именно ударная волна), но, возможно, и с расхождением волны согласно (2). 

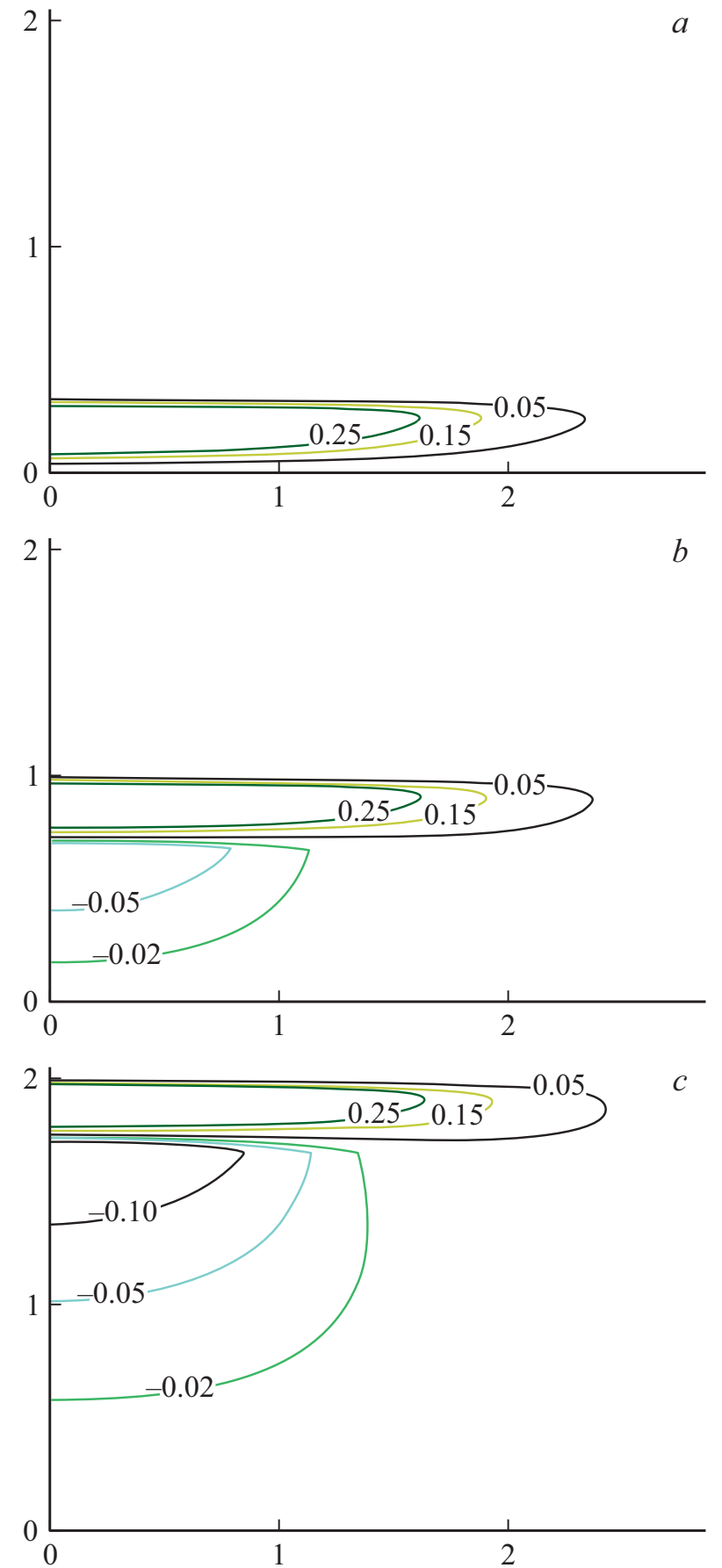

Рис. 5. Изолинии напряжения в упругой волне при различных значениях $t: a-0.34$ (т. е. сразу после окончания импульса давления), $b-1.0$ и $c-2.0$. Горизонтальная ось соответствует радиальной координате, вертикальная - оси $z$.

\section{Заключение}

Итак, мы показали, что загадочный характер разрушения ПММА и других нехрупких полимеров под воздействием возбуждаемого РЭП упругого возмущения может быть объяснен достаточно простым и наглядным образом. При этом стоит отметить, что у ПММА и эпок- сидных смол наблюдаются схожие особенности релаксации (см., например, [19]). В более хрупких материалах механизмы релаксации напряжений могут существенно отличаться [5,6], и растрескивание не столь кардинально связано с высокими значениями $\sigma<0$. За счет этого и различаются картины на рис. 2 и 3. В принципе эффект „генерации“ области растяжения должен был бы наблюдаться в расчетах [20], где основное внимание уделено „полномасштабному“ описанию упругой среды с ненулевыми значениями и $K$, и $G$ (без учета превышения предела текучести), но в тексте о нем ничего не говорится. Хотя эволюция волны в этом случае сложнее, и скорость среды в ней уже не потенциальна, но само явление, как сказано во Введении, на качественном уровне устойчиво к таким возмущениям. Возможно, дело в том, что авторы сосредоточились на моделировании разрушения хрупкого стекла, растрескивающегося и при $\sigma>0$, а потому не обратили внимания на, казалось бы, малые отрицательные значения напряжения.

\section{Финансирование работы}

Работа выполнена при поддержке гранта НИЦ „Курчатовский институт“ (приказ № 2073 от 09 октября 2020 г.).

\section{Конфликт интересов}

Авторы заявляют, что у них нет конфликта интересов.

\section{Список литературы}

[1] Б.А. Демидов, В.П. Ефремов, М.В. Ивкин, В.А. Петров, А.Н. Мещеряков. Поверхность, 8, 55 (2008).

[2] Б.А. Демидов, В.П. Ефремов, В.А. Петров, А.Н. Мещеряков. Поверхность, 9, 18 (2009).

[3] С.А. Абросимов, А.П. Бажулин, В.В. Воронов, И.К. Красюк, П.П. Пашинин, А.Ю. Семенов, И.А. Стучебрюхов, К.В. Хищенко, В.И. Черномырдин. ДАН, 442 (6), 752 (2012). [S.A. Abrosimov, A.P. Bazhulin, V.V. Voronov, I.K. Krasyuk, P.P. Pashinin, A.Yu. Semenov, I.A. Stuchebryukhov, K.V. Khishchenko. Dokl. Phys., 57 (2), 64 (2012).]

[4] S.F. Gnyusov, V.P. Rotshtein, A.E. Mayer, V.V. Rostov, A.V. Gunin, K.V. Khishchenko, P.R. Levashov. Int. J. Fracture, 199 (1), 59 (2016).

[5] Б.М. Бартенев. Релаксационные свойства полимеров (Химия, М., 1992)

[6] И.И. Перепечко. Акустические методы исследования полимеров (Химия, М., 1973)

[7] Б.А. Демидов, В.Л. Ефремов, Ю.Г. Калинин, В.А. Петров, С.И. Ткаченко, К.В. Чукбар. ЖТФ, 82 (3), 94 (2012).

[B.A. Demidov, V.P. Efremov, Y.G. Kalinin, V.A. Petrov, S.I. Tkachenko, K.V. Chukbar. Tech. Phys., 57 (3), 405 (2012). DOI: $10.1134 / \mathrm{S} 106378421203005 \mathrm{X}$

[8] Б.А. Демидов, Е.Д. Казаков, А.А. Курило. ВАНТ. Серия: Термоядерный синтез, 40 (2), 73 (2017).

[9] Г.И. Канель, С.В. Разоренов, А.В. Уткин, В.Е. Фортов. Ударно-волновые явления в конденсированных средах (Янус-К, М., 1996) 
[10] Я.Б. Зельдович, Ю.П. Райзер. Физика ударных волн u высокотемпературных гидродинамических явлений (Наука, М., 1966)

[11] S.P. March. Lasl Shock Hugoniot Data (University of California Press, Berkley, 1980)

[12] Б.А. Демидов, Е.Д. Казаков, Ю.Г. Калинин, Д.И. Крутиков, А.А. Курило, М.Ю. Орлов, М.Г. Стрижаков, С.И. Ткаченко, К.В. Чукбар, А.Ю. Шашков. ПТЭ, 3, 90 (2020). DOI: 10.31857/S003281622003009X [B.A. Demidov, E.D. Kazakov, Y.G. Kalinin, D.I. Krutikov, A.A. Kurilo, M.Y. Orlov, M.G. Strizhakov, S.I. Tkachenko, K.V. Chukbar, A.Y. Shashkov. Instrum. Exp. Tech., 63, 370 (2020). DOI: $10.1134 / \mathrm{S} 0020441220030094]$

[13] С.С. Ананьев, Г.А. Багдасаров, В.А. Гасилов, С.А. Данько, Б.А. Демидов, Е.Д. Казаков, Ю.Г. Калинин, А.А. Курило, О.Г. Ольховская, М.Г. Стрижаков, С.И. Ткаченко. Физика плазмы, 43 (7), 608 (2017). [S.S. Ananyev, G.A. Bagdasarov, V.A. Gasilov, S.A. Dan'ko, B.A. Demidov, E.D. Kazakov, Y.G. Kalinin, A.A. Kurilo, O.G. Ol'hovskaya, M.G. Strizhakov, S.I. Tkachenko. Plasma Phys. Rep., 43, 726 (2017). DOI: $10.1134 / \mathrm{S} 1063780 \mathrm{X} 17070029]$

[14] Б.А. Демидов, В.П. Ефремов, Е.Д. Казаков, Ю.Г. Калинин, С.Ю. Метелкин. ПТЭ, 2, 96 (2016). DOI: 10.7868/S003281621602004X [B.A. Demidov, V.P. Efremov, E.D. Kazakov, Y.G. Kalinin, S.Y. Metelkin. Instrum Exp Tech., 59, 258 (2016). DOI: 10.1134/S0020441216020044]

[15] Л.Д. Ландау, Е.М. Лифшиц. Гидродинамика (Физматлит, М., 2001)

[16] Дж. Лайтхилл. Волны в жсидкостях (Мир, М., 1981) [Пер. с англ. M.J. Lighthill. Waves in Fluids (Cambridge University Press, 2001)]

[17] Б.Б. Кадомцев. Коллективные явления в плазме (Наука, M., 1988)

[18] А.П. Прудников, Ю.А. Брычков, О.И. Маричев. Интегралы и ряды. Специальные функции (Физматлит, М., 2003), т. 2, c. 168.

[19] В.М. Мочалова, А.В. Уткин, А.В. Павленко, С.Н. Малюгина, С.С. Мокрушин. ЖТФ, 89 (1), 126 (2019).

[V.M. Mochalova, A.V. Utkin, A.V. Pavlenko, S.N. Malyugina, S.S. Mokrushin. Tech. Phys., 64 (1), 100 (2019).

DOI: $10.1134 / \mathrm{S} 1063784219010225]$

[20] В.А. Гасилов, А.С. Грушин, А.С. Ермаков, И.Б. Петров, О.Г. Ольховская. Математическое моделирование, 30 (7), 61 (2018). DOI: 10.31857/S023408790000575-6 